\title{
Extensive Purpura and Necrosis of the Leg
}

Michael Musharbash, MD; Lida Zheng, MD; Lauren Guggina, MD

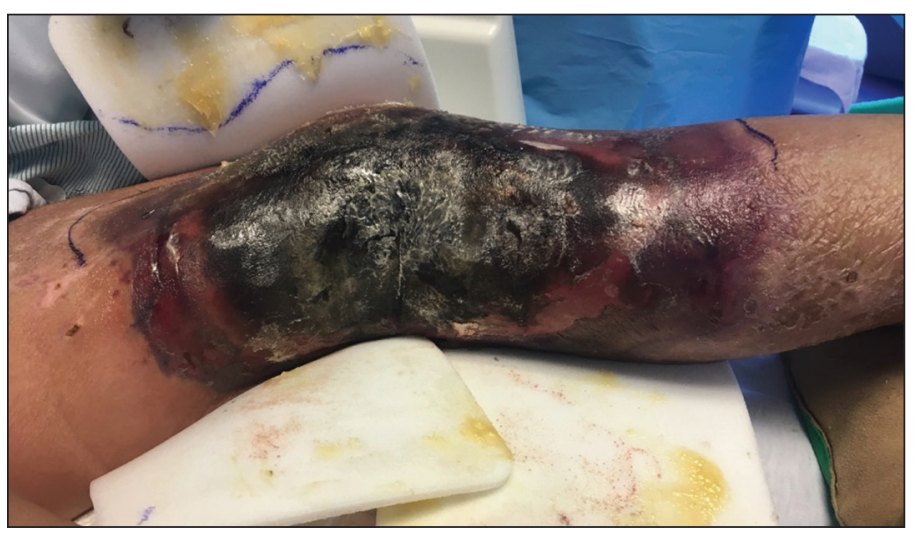

A 57-year-old woman presented with expanding purpura on the left leg of 2 weeks' duration following a recent hematopoietic stem cell transplant for refractory diffuse large B-cell lymphoma. Prior to dermatologic consultation, the patient had been hospitalized for 2 months following the transplant due to Clostridium difficile colitis, Enterococcus faecium bacteremia, cardiac arrest, delayed engraftment with pancytopenia, and atypical hemolytic uremic syndrome with acute renal failure requiring hemodialysis and treatment with eculizumab. Her care team in the hospital initially noticed a small purpuric lesion on the posterior aspect of the left knee. The patient subsequently developed persistent fevers and expansion of the lesion, which prompted consultation of the dermatology service. Physical examination revealed a $22 \times 10-\mathrm{cm}$, rectangular, indurated, purpuric plaque with central dusky, violaceous to black necrosis with superficial skin sloughing and peripheral dusky erythema extending from the inner thigh to the lower leg. The left distal leg felt cool, and both dorsalis pedis and posterior tibial pulses were absent. Laboratory test results revealed neutropenia and thrombocytopenia

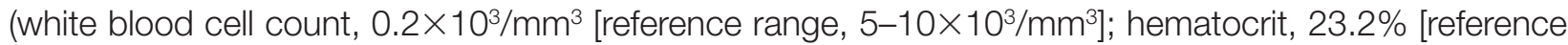
range, 41\%-50\%]; platelet count, $105 \times 10^{3} / \mu \mathrm{L}$ [reference range, $150-350 \times 10^{3} / \mu \mathrm{L}$ ]). A punch biopsy was performed.

\section{WHAT'S THE DIAGNOSIS?}
a. disseminated aspergillosis
b. disseminated intravascular coagulation
c. disseminated mucormycosis
d. purpura fulminans
e. pyoderma gangrenosum

\footnotetext{
From the Department of Dermatology, Northwestern Memorial Hospital, Chicago, Illinois. 


\section{THE DIAGNOSIS:}

\section{Disseminated Mucormycosis}

$\square$ istopathologic examination of a $6-\mathrm{mm}$ punch biopsy of the edge of the lesion revealed numerous intravascular, broad, nonseptate hyphae in the deep vessels and perivascular dermis that stained bright red with periodic acid-Schiff (Figure). Acid-fast bacilli and Gram stains were negative. Tissue culture grew Rhizopus species. Given the patient's overall poor prognosis, her family decided to pursue hospice care following this diagnosis.

Mucormycosis (formerly zygomycosis) refers to infections from a variety of genera of fungi, most commonly Mucor and Rhizopus, that cause infections primarily in immunocompromised individuals. ${ }^{1}$ Mucormycosis infections are characterized by tissue necrosis that results from invasion of the vasculature and subsequent thrombosis. The typical presentation of cutaneous mucormycosis is a necrotic eschar accompanied by surrounding erythema and induration. ${ }^{2}$ Diagnosis is based on clinical suspicion, requiring additional testing with skin biopsy and tissue cultures for confirmation.

Cutaneous infection is the third most common presentation of mucormycosis, following rhinocerebral and pulmonary involvement. ${ }^{1}$ Although rhinocerebral and pulmonary infections normally are caused by inhalation of spores, cutaneous mucormycosis typically is caused by local inoculation, often following skin trauma. ${ }^{2}$ The skin is the most common location of iatrogenic mucormycosis, often from skin injury related to surgery, catheters, and adhesive tape. ${ }^{3}$ Most patients with cutaneous mucormycosis have underlying conditions such as hematologic malignancies, diabetes mellitus, or immunosuppression. ${ }^{1}$ However, outbreaks have occurred in immunocompetent patients following natural disasters. ${ }^{4}$ Cutaneous mucormycosis disseminates in $13 \%$ to $20 \%$ of cases in which mortality rates typically exceed $90 \% .^{1}$

Treatment consists of prompt surgical debridement and antifungal agents such as amphotericin B, posaconazole, and isavuconazonium sulfate. ${ }^{1}$ Our patient had multiple risk factors for infection, including hematopoietic stem cell transplantation, prolonged neutropenia, and treatment with eculizumab, a monoclonal antibody against C5 that blocks the terminal complement cascade. Eculizumab has been associated with increased risk for meningococcemia, ${ }^{5}$ but the association with mucormycosis is rare. We highlight the importance of recognizing and promptly diagnosing cutaneous mucormycosis given the difficulty of treating this disease and its poor prognosis.

Disseminated aspergillosis demonstrates septate rather than nonseptate hyphae on biopsy. Disseminated intravascular coagulation and purpura fulminans may

be associated with thrombocytopenia but demonstrate thrombotic microangiopathy on biopsy. Pyoderma gangrenosum demonstrates neutrophilic infiltrate on biopsy.

\section{REFERENCES}

1. Roden MM, Zaoutis TE, Buchanan WL, et al. Epidemiology and outcome of zygomycosis: a review of 929 reported cases. Clin Infect Dis. 2005;41:634-653.

2. Petrikkos G, Skiada A, Lortholary O, et al. Epidemiology and clinical manifestations of mucormycosis. Clin Infect Dis. 2012;54(suppl 1):S23-S34.

3. Rammaert B, Lanternier F, Zahar JR, et al. Healthcare-associated mucormycosis. Clin Infect Dis. 2012;54(suppl 1):S44-S54.

4. Neblett Fanfair R, Benedict K, Bos J, et al. Necrotizing cutaneous mucormycosis after a tornado in Joplin, Missouri, in 2011. N Engl J Med. 2012;367:2214-2225.

5. McNamara LA, Topaz N, Wang $X$, et al. High risk for invasive meningococcal disease among patients receiving eculizumab (Soliris) despite receipt of meningococcal vaccine. MMWR Morb Mortal Wkly Rep. 2017;66:734-737 